\title{
The discrete analysis of the tissue biopsy images with metamaterial formalization: identifying tumour locus
}

\author{
Tatjana Gric, Sergei G. Sokolovski, Alexander G. Alekseev, Andrian V. Mamoshin, Andrey Dunaev, \\ Edik U. Rafailov
}

\begin{abstract}
Herein, we develop an enhanced and automated methodology for detection of the tumour cells in fixed biopsy samples. Metamaterial formalism (MMF) approach allowing recognition of tumour areas in tissue samples is enhanced by providing an advanced technique to digitize mouse biopsy images. Thus, a colour-based segmentation technique based on the $K$ means clustering method is used allowing for a precise segmentation of the cells composing the biological tissue sample. Errors occurring at the tissue digitization steps are detected by applying MMF. Doing so, we end up with the robust, fully automated approach with no needs of the human intervention, ready for the clinical applications. The proposed methodology consists of three major steps, i. e. digitization of the biopsy image, analysis of the biopsy image, modelling of the disordered metamaterial. It is worthwhile mentioning, that the technique under consideration allows for the cancer stage detection. Moreover, early stage cancer diagnosis is possible by applying MMF.
\end{abstract}

Index Terms - cancer; metamaterial.

\section{INTRODUCTION}

Cancer is one of the leading reasons of death worldwide. In this relation, its diagnosis is crucial to start therapies [1]. The apoptosis of cancer cells plays a pivotal role in shaping of organs in tandem with cell proliferation, regulation, and the removal of defective as well as excessive cells in immune system [2-4]. Consequently, it is important to create precise recognition technologies that can confirm if the biological tissue is cancerous.

The key feature of the cancer affected tissue is the presence of the glioma cells in the sample. Current approaches in distinguishing healthy-tumour areas mainly focus on fluorescent, immunological, and morphological differences

Tatjana Gric is with Department of Electronic Systems, Vilnius Gediminas Technical University, Vilnius, Lithuania; Aston Institute of Photonic Technologies, Aston University, Birmingham B4 7ET, UK; Semiconductor Physics Institute, Center for Physical Sciences and Technology, Vilnius, Lithuania.

Sergei G. Sokolovski is with Aston Institute of Photonic Technologies, Aston University, Birmingham B4 7ET, UK; Laboratory of Optics and Dynamics of the Biological Systems, School of Physics, Novosibirsk State University, Novosibirsk, Russia.

Alexander G. Alekseev is with Clinical Multidisciplinary Center for Medical Care for Mothers and Children named after Z.I. Krugloy, Orel, Russia; highlighted by different staining, intrinsic autofluorescence and decretive microscopy methods rather than on the "metamaterial formalism (MMF)" of the diseases. Fluorescence microscopy and immunofluorescence methods stand for as the main approaches aiming to identify and analyze tumor cells by imaging specific markers that depend on the phenotypes of the tumor cells [5]. Nevertheless, the former seems to be quite challenging issue. Specifically, manual calculations and assessment by qualified specialists are requested. The former are prone to develop biased criteria and fatigue over time. Doing so, one may face the erroneous conclusions based on data. An implemented framework that can handle high data throughput in both, the hardware and software is needed seeking for the reliable reproducibility and deterministic interpretation. Thus, a drastic minimization of the human intervention can be achieved [6]. The MMF is an enhanced toll providing a fertile ground for the design of the entirely computerized system. It is worthwhile mentioning, that in this case human involvement is not needed. Thus, biological tissue is treated as the disordered metamaterial model. Moreover, the former approach allows to significantly reduce possibility of errors that are possible at the tissues digitizing stage. The MMF allows to calculate the critical minimum of the glioma cells in the biopsy sample thus recognizing developing tumour that might help to prevent the early stage cancer. It is becoming possible to "see the metamaterial features" in the biological tissue samples with the development of the effective medium approximation techniques. The advantage of this approach lies in its ability defining the biological tissue as the metamaterial structure.

A large knowledgebase has been created thanks to the recent studies of metamaterials [7-9]. Thus, the significance of the MMF for dealing with biological challenges [10-12] is clearly observed. Sensing, chemical and biomedical applications are

Department of Anatomy, Operative Surgeons and Disaster Medicine, Orel State University, Orel, Russia.

Andrian V. Mamoshin is with Research \& Development Center of Biomedical Photonics, Orel State University, Orel, Russia; Orel Region Clinical Hospital, Orel, Russia.

Andrey Dunaev is with Research \& Development Center of Biomedical Photonics, Orel State University, Orel, Russia.

Edik U. Rafailov is with Aston Institute of Photonic Technologies, Aston University, Birmingham B4 7ET, UK; Aston Institute of Photonic Technologies, Aston University, Birmingham B4 7ET, UK and with Peter the Great St. Petersburg Polytechnic University, St. Petersburg, Russia. 
possible thanks to the recent studies of electromagnetic radiation in $\mathrm{THz}$ frequency range. $\mathrm{THz}$ metamaterials are composed of periodically arranged sub-wavelength metal structures. It should be stressed that biological tissue can be treated form the perspectives of the disordered metamaterials [13-15] theory in terms of the distribution of the cells composing the structures. Doing so, the MMF is widely applicable to treat the biological processes.

During past years the calculation of effective permittivity of biological tissue nonlinear characteristics in frequency domain being serious challenge received tremendous attention [16, 17]. To the best of our knowledge, there is no study proposing application of MMF aiming to conclude if the sample is cancerous. This work evaluates the effective permittivity of biological tissue giving a full credit to the MMF. Here we imply a theoretical study of effective permittivity in biomaterials deliberately for the mouse models. Recognition of cancerous tissues is possible because of the precise determination of the effective permittivity of the tissue samples.

The rapid development of machine learning and especially deep learning allows for the significant improvement of the accuracy of cancer screening [18]. Machine learning algorithms can assist in analysing large amounts of data and solving complicated problems in a precise and fast way $[19,20]$. It can extensively assist physicians to improve the accuracy and efficiency in diagnosing cancer, choosing appropriate therapeutic approaches and predicting long-term outcomes. Artificial intelligence (AI) [21] usually deploys a subset of machine learning technics that identify patterns in data and train a machine how to learn [22]. Giving the full credit to all the advantages possessed by the developed methodologies, the main their drawback should be considered. The former appears due to the tremendous computer resources needed to deal with challenges. The application of the MMF will allow for a more efficient detection and identification of cancerous samples with much less computer resources due to the applied effective medium approximation techniques.

At the first time to our knowledge, here we manage to formalise the biological tissues as a metamaterial helping in discriminating non and cancerous areas in the brain tissues. The obtained effective permittivity values were dependent on various factors, including the amount of different cell types in the sample and their distribution. Moreover, the recognition of the cancerous areas will be proposed based on their effective medium properties. The MMF can make a dramatic impact on the development of methodological approaches seeking for a precise identification of pathological tissues and would allow for more effective detection of cancer-related changes.

To our best knowledge, there is no study applying MMF for recognising the cancerous tissues. In the research we will deploy a theoretical analysis of the effective permittivity of materials aiming to explore the acceptability of the models [35] to treat biological tissues by providing a significant enhancement at the tissue digitization stage.

\section{MMF APPROACH}

The structure of the fully automated MMF approach is in the form of several steps, i. e. digitization of the biopsy image, digitized image analysis, modelling of the disordered metamaterial (Fig. 1). For understanding whether the examined biological sample is non-cancerous, it is required analyse it in terms of the effective medium approximation [23]. The applicability of the MMF becomes feasible when the distribution of the cells is clearly identified in the sample under investigation. Consequently, we will apply image digitizer techniques seeking for the identification of all morphological and functional cell types and their exact positions. Thus, the coordinates of the mouse brain biological tissue cells will be clearly depicted in samples. It is of particular importance to have a deep insight at the filling ratio of each type of the cells identified in the in the histograms aiming to treat the obtained biological tissue image form the perspective of the MMF. Furthermore, the optical properties of the cells should be described by Debye and Cole-Cole models [24]. Thus, the complex dielectric spectrum of all cell types is provided in $\varepsilon(\omega)$ space. A schematic illustration of the MMF-based approach is presented in Fig. 1. The biopsy image is to be digitized at the initial stage of the data processing. It might be performed by choosing one of three approaches, i. e. K-means clustering, differential evolution algorithm or Digitizeit software. At the next algorithmic step the obtained information is analysed in creating defined cell databases. Moreover, in further proceeding the application of the disordered metamaterial model, cells distribution within biopsy images should be accurately positioned. Particularly, we are interested in assessing the cell geometry, their shapes and sizes and cell location coordinates in the sample. These parameters be used in full can finally create the disordered metamaterial model. Aiming in proceeding further with the accomplished model, cells permittivities are identified seeking for a homogenization of the structure in use by means of the effective medium approximation. All the presented steps might be fully automated by employing artificial intelligence approach. 


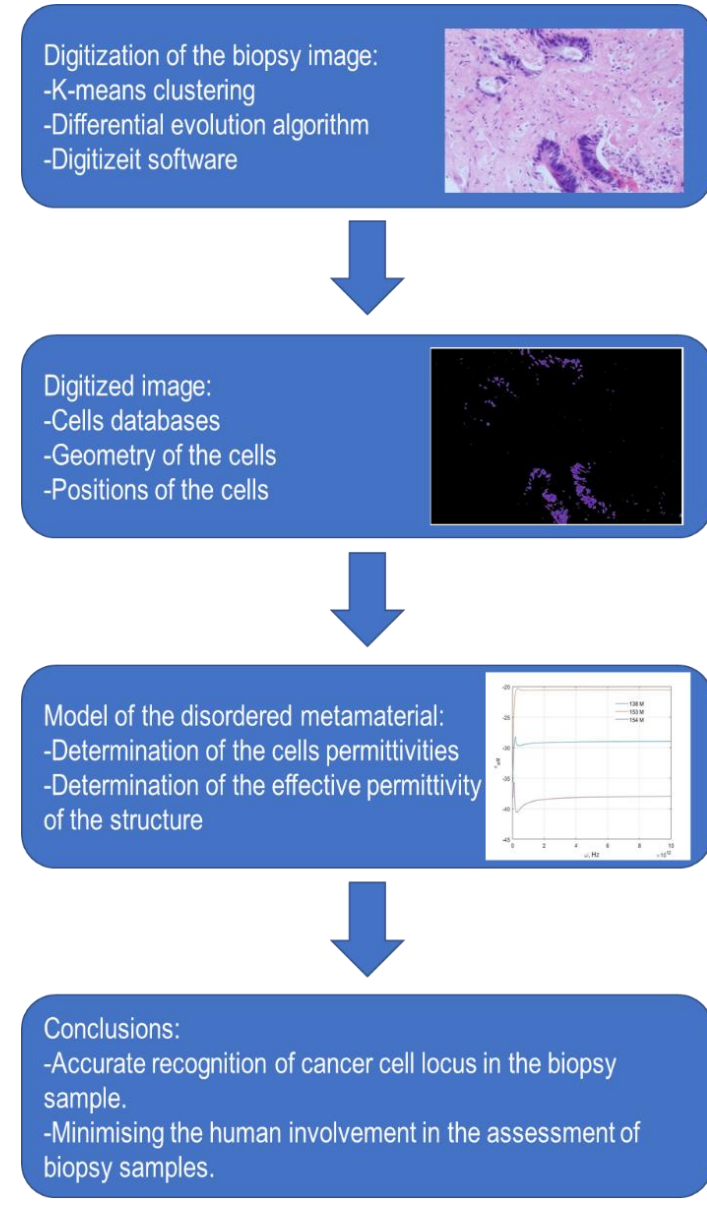

Figure 1. A scheme of the metamaterial-based algorithm approach in distinguishing precise location of the tumour

\subsection{Dataset composition} locus

Work carried out at the department of interventional radiology of Orel Regional Clinical Hospital (Orel, Russia). The study was also approved by the Ethics committee of Orel State University (record of the meeting No. 14 of 24.01.2019) and was carried out in accordance with the 2013 Declaration of Helsinki by the World Medical Association. Patients scheduled for a regular needle biopsy for a suspected liver malignancy were recruited for study participation. The lesions had to be safely accessible. Patients at increased risk of bleeding were excluded. After receiving the description of the protocol, the patients signed informed consent indicating their voluntary willingness to participate in the study. The experiments were conducted under established protocols. The measurements were performed during standard needle biopsy procedure in 15 patients (6 male, 9 female) with supposed liver cancer, the median age was $66 \mathrm{yr}$ (range 45 to $78 \mathrm{yr}$ ).

The procedure was carried after the proper skin disinfection and injection of a local anesthetic (2\% lidocaine hydrochloride solution). At each selected site, the surgeon inserted a $17.5 \mathrm{G}$ needle $1.3 \mathrm{~mm}$ into the liver tissue. We followed by the standard procedure of the biopsy sampling and histological examination. The tumor tissue samples were fixed with $10 \%$ neutral-buffered formalin, dehydrated, and embedded in paraffin. Approximately 5 - $\mu \mathrm{m}$-thick sections were stained by haematoxylin and eosin method according to standard procedures. Histological specimen was examined with a Leica DM2000 microscope at a magnification of $\times 100 ; \times 200$; $\times$ 400.The resulting tissue slices were examined by light microscopy by an experienced pathologist. Digital images of various fields of view were obtained using a Leica DFC295 camera, 4 micrographs at each magnification.

\section{$2.2 \mathrm{~K}$-means clustering}

$\mathrm{K}$-means is extensively applied clustering approach aiming to partition data into $\mathrm{k}$ clusters $[25,26]$. Clustering is an approach which is based on collecting two types of the data points, with analogous feature vectors into a single cluster and with different feature vectors into other clusters. Basically, feature space selection is a key point in K-means clustering segmentation. An RGB colour map comprises R, G, and B values for each item. The RGB colour space is further converted to a CIELab colour model (L*a*b*) [27] aiming to extract significant features aiming to benefit from the clustering approach. The $L^{*} a * b *$ space involves a luminosity layer $L^{*}$, a chromaticity-layer $\mathrm{a}^{*}$, which indicates where colour falls along the red-green axis, and chromaticity-layer $b^{*}$, which indicates where the colour falls along the blue-yellow axis. Both the $a^{*}$ and $b^{*}$ layers comprise all mandatory colour information. This way allows to classify the colours in the $\mathrm{a}^{*} \mathrm{~b}$ * space by means of K-means clustering. When the clustering process is fulfilled, the cluster comprising an area of interest is designated as the primary segment. To remove the pixels which are not related to the selected cluster, histogram clustering may be applied by luminosity feature $\mathrm{L}^{*}$ aiming to derive the final segmented result.

\subsection{Differential evolution algorithm}

The automatic detection of cancer cells still stands for as an unresolved problem in medical imaging. The examination of the obtained images has stimulated an unprecedented interest of the researchers from the areas of medicine and computer vision. It is worthwhile noting, that cancer cell can be approximated by an ellipsoid form. Doing so, one may successfully apply an ellipse detector algorithm aiming to identify such elements. We have applied the automatic recognition of the cancerous cells implanted into complex and cluttered smear images. Thus, the complete process is treated as a multi-ellipse detection task. The detection task is transformed into an optimization issue that represent candidate ellipses. The former procedures are dictated by the approach, which is based on the DE algorithm. An objective function stands for to evaluate if such candidate ellipses are present in the edge map of smear image. Guided by the values of such function, the set of encoded candidate ellipses (individuals) are evolved using the $\mathrm{DE}$ algorithm so that they can fit into the WBC which are enclosed within the edge map of the smear image.

\subsection{Digitizeit software}

For the sake of identification the position of cells comprising the tissue sample, segmentation algorithms are needed to locate the anticipated region boundaries and label all cells within a single continuous boundary as belonging to one region. 
Datasets of the cell types are designed based on the geometry, colour and size of the cells. Doing so, a variety of measurements can be made using established methods [28]. One may take an advantage from this approach by the reduced analysis time [29]. We apply https://www.digitizeit.de/ software to recognize the cell sets forming the considered tissue. Doing so, one may benefit from the faster operation time in comparison with OpenCV [30] for C\# in Microsoft Visual Studio. On the contrary, the identification algorithm based on Otsu thresholding will require significant computer resources. The former is known is a threshold algorithm for image segmentation [31]. To conclude, our applied technique has a faster run time and requires less computer resources than cancer cell detection methods implemented with OpenCV [30] for C\# in Microsoft Visual Studio. It is a perfect tool aiming to obtain a digitized view of the biopsy sample under consideration, that will be treated as a disordered metamaterial media at the later stages of consideration. It is worthwhile noting, that fluorescence microscopy and immunofluorescence methods are used as the conventional techniques for tumor cells detection and assessment. Aiming to fulfil these procedures imaging of specific markers that are dramatically affected by the phenotypes of the tumor cells is needed [32]. Nevertheless, application of such approaches is quite challenging and complicated issue. Seeking for the reliable reproducibility and deterministic interpretation implementation of a framework that can handle high data throughput in both, the hardware and software is of particular importance. Thus, human intervention can be significantly reduced [33].

\subsection{Calculation of the effective permittivity of the disordered metamaterial model}

The MMF is a perfect tool aiming to create the entirely computerized system. It is worthwhile mentioning, that in this case human involvement is not needed. The techniques created allow to calculate the critical limit of the glioma cells which allow to delimitate the tumour boundaries that in turn give opportunity to identify cancer at the early stages [35]. As saying whether the biological tissue is non-cancerous, biopsy sample image is to be analysed using the effective medium approximation [34]. One may apply the MMF method if the distribution of cells in the considered medium is clearly identified in terms of the distribution of the cells in the sample. Doing so, image digitizer techniques are applied aiming to have a deep insight in to the exact coordinates of the cell sets combining the sample. It should be mentioned, that the coordinates of the glioma, glia and neuronal cells were clearly demonstrated in [35]. Aiming to apply the MMF approach, the optical properties of the cells should be characterized by Debye and Cole-Cole models [36]. In this relation, the complex dielectric spectrum for all cell types is shown in $\varepsilon(\omega)$ space.

Aiming to have a deep insight into the nature of the biopsy sample under investigation, creation of the permittivity model for brain tissue biopsies is of particular importance. A typical theory attained on the basis of empirical formula [36] is Debye model. Other widely applied permittivity models based on binary mixtures do not account for the frequency domain, for instance, the Bottcher-Bordewijk model [37], Maxwell-Garnett formula [38], Bruggeman formula [39] and Hanai formula [40]. It should be mentioned, that the Bottcher-Bordewijk model allows for a feasible prediction of the permittivity of the medium [41], i. e. effective permittivity of the biological tissue sample. The model is described as follows:

$$
\frac{3 \varepsilon_{1}}{2 \varepsilon_{\text {eff }}+\varepsilon_{1}} f_{1}+\frac{3 \varepsilon_{2}}{2 \varepsilon_{\text {eff }}+\varepsilon_{2}} f_{2}=1
$$

In this case cell structure is of a random nature possessing some predictable average properties such as cell size and cell distribution density. It can be modelled by an aggregated of randomly distributed spherical shells. This model can be applied aiming to describe the two constituent materials. Another famous model put forward by Skipetrov [42] can be applied dealing with the two-phase system. The former is found by solving Eq. (1) with respect to $\varepsilon_{e f f}$, i. e.

$$
\varepsilon_{\text {eff }}=\frac{3 \varepsilon_{1} f_{1}}{4}-\frac{\varepsilon_{2}}{4}-\frac{\varepsilon_{1}}{4}+\frac{3 \varepsilon_{2} f_{2}}{4}-A,
$$

Here

$$
A=\frac{\sqrt{9 \varepsilon_{1}^{2} f_{1}^{2}-6 \varepsilon_{1}^{2} f_{1}+\varepsilon_{1}^{2}+18 \varepsilon_{1} \varepsilon_{2} f_{1} f_{2}+6 \varepsilon_{1} \varepsilon_{2} f_{1}+6 \varepsilon_{1} \varepsilon_{2} f_{2}-2 \varepsilon_{1} \varepsilon_{2}+9 \varepsilon_{2}^{2} f_{2}^{2}-6 \varepsilon_{2}^{2} f_{2}+\varepsilon_{2}^{2}}}{4} \varepsilon_{1}
$$

denotes the permittivity of the neuron cells, $\varepsilon_{2}$ - of the glioma cells, $f_{1}$ and $f_{2}$ are the volume fractions of each cell type accordingly. Furthermore, $\varepsilon_{\mathrm{eff}}$ is the effective permittivity characterizing effective properties of the medium. The dielectric properties $\varepsilon_{k}(\omega), k=1,2$, are obtained by means of the Cole Cole spectral function [43].

$$
\varepsilon_{k}=\varepsilon_{k}(\infty)+\frac{\varepsilon_{k}(0)-\varepsilon_{k}(\infty)}{1+\left(i \omega \tau_{k}\right)^{1-\alpha_{k}}}+\frac{\sigma_{k}}{i \varepsilon_{0} \omega}
$$

with $\varepsilon_{k}(0)$ - static dielectric permittivity, $\varepsilon_{k}(\infty)$ - high frequency dielectric permittivity, $\tau_{k}-$ relaxation time, $\alpha_{k}-$ distribution parameter, $\sigma_{k}-\mathrm{DC}$ conductivity. On the basis of the described methodology, the dependences of the effective permittivity upon frequency for different cases (differences in the volume fraction $f$, permittivities of the building blocks (cells)) have been attained. It should be mentioned, that the effective permittivity was obtained theoretically by means of Skipetrov model (Eq. (2)). The spatially dependent volume fraction has been extracted from the digitized images and included into the effective medium model (Eq. (2)). The former assumes the prior knowledge of the frequency-dependent permittivity of the building block (cells) characterized by (Eq. (3)). The outstanding feature of the described model is the possibility of the extraordinary nature of the effective permittivity. This is illustrated by the peak of effective permittivity curve in the certain case at the frequency range $(0$ $10 \mathrm{THz}$ ) [35] if the total amount of the glioma cells in the sample exceeds $5 \%$. 


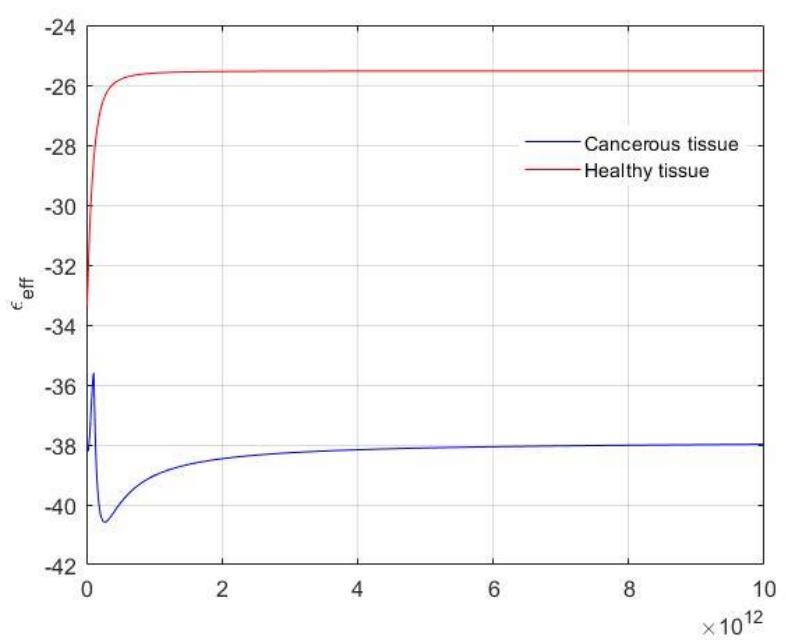

Figure 2. Example of the dependence of the effective permittivity upon frequency for the case of mouse brain cancer (blue) and healthy tissue (red) [35]

As it was discovered in [35] (Fig. 2) effective permittivity values are negative. The former takes place due to the characterization of the cells by means of the Cole Cole spectral function (Eq. 3). It should be mentioned, that the biological sample described by the metamaterial [35] experiences extraordinary behaviour. The former takes place because of the certain amount of glioma cells exceeding the certain limit, i. e. $5 \%$. It is worthwhile mentioning that the minimum detectable tumor area is $0.3 \mu \mathrm{m}^{2}$. It can be concluded, that the tissue is cancerous because of the extraordinary behaviour of the effective permittivity curve. It is clearly observed, that there is a certain peak at the frequency around $0.1 \mathrm{THz}$. Consequently, extraordinary nature occurs, when the amount of unhealthy specimen(s) in the sample exceeds the certain limit. Based on the obtained results, the high amplitude peak is found in the case of $154 \mathrm{M}$ [35] sample. The former serves as the evidence of the presence of the highly cancerous tissue zones [35] in the sample. The higher amplitude of $\varepsilon_{\text {eff }}$ corresponds to the later stage cancer. In this, stage refers to the extent of cancer and is based on factors such as dimensions o the tumour and possibility for it to spread. Aiming to approbate created algorithm the randomly chosen tissue samples (25 instances) have been considered. The described metamaterial approach allows for a precise recognition of the healthy and cancerous tissues. The obtained results have been approved by the histological analysis with $100 \%$ success rate [35].

\section{CNN-BASED ANALYSIS OF LIVER BIOPSY IMAGES AFTER MMF APPLIED}

The digitized images of the mouse liver biopsies are presented in Fig. 3. The staining procedure for histological preparations was carried out under standard conditions. The sections were of the same thickness, certified dyes were used, the same staining time and temperature were applied. The internal quality control process was handled by the histologist and pathologist independently. Some visual discrepancy in color is due to different increases in preparations. Figure $3 \mathrm{~A}$ shows liver tissue. The degree of staining of the cytoplasm here is somewhat different from Figure $3 \mathrm{D}$, but fully corresponds to the picture that the researcher sees in the microscope, which is important. Figure $3 \mathrm{D}$ shows more eosinophilic staining in areas of connective tissue (between tumor cells), there are no such areas in Figure 3 A.

All the types of the cells have been recognized by K-means clustering approach. The segmentation aims to classify the cells into different groups. By calculating effective permittivity plots after careful investigation of the cells permittivities (Eq. 3), MMF approach [35] allows to detect cancerous locus within the biopsy images.

In the proposed K-means clustering method, a liver biopsy image consists of regions that represent cancer cells, the background and the connective tissue. In these data sources, visual judgements suggest three primary clusters, when $k=3$. Figs. 3B-H show the image labelled by cluster index from the $\mathrm{K}$-means process for different kinds of feature vectors. Using index labels, we can separate objects in the liver image by three colours: white, blue, pink. The final segmentation results generated by histogram clustering are shown in Figs. 3B-H.

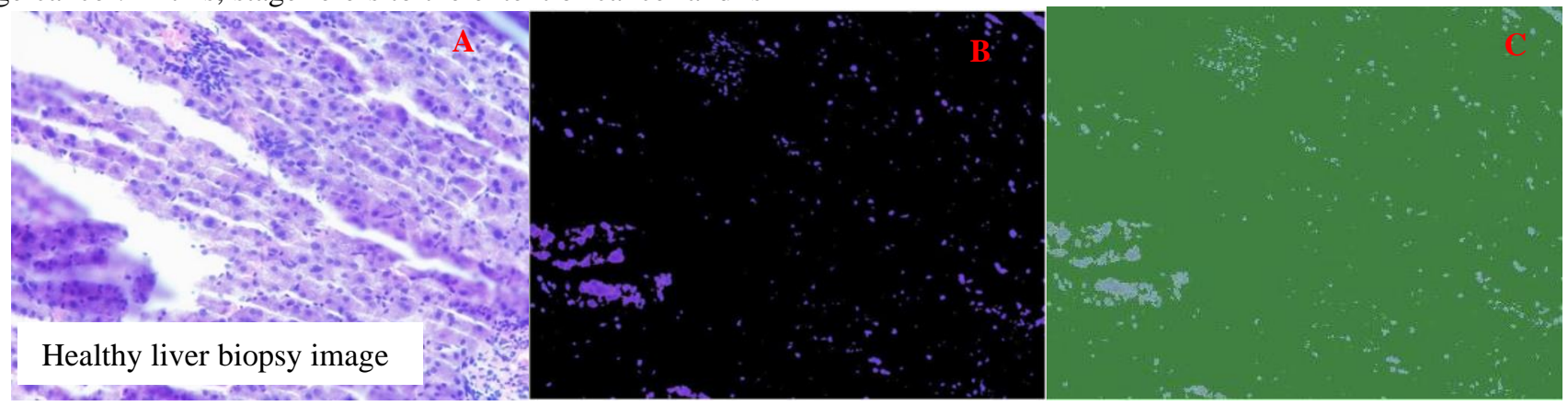




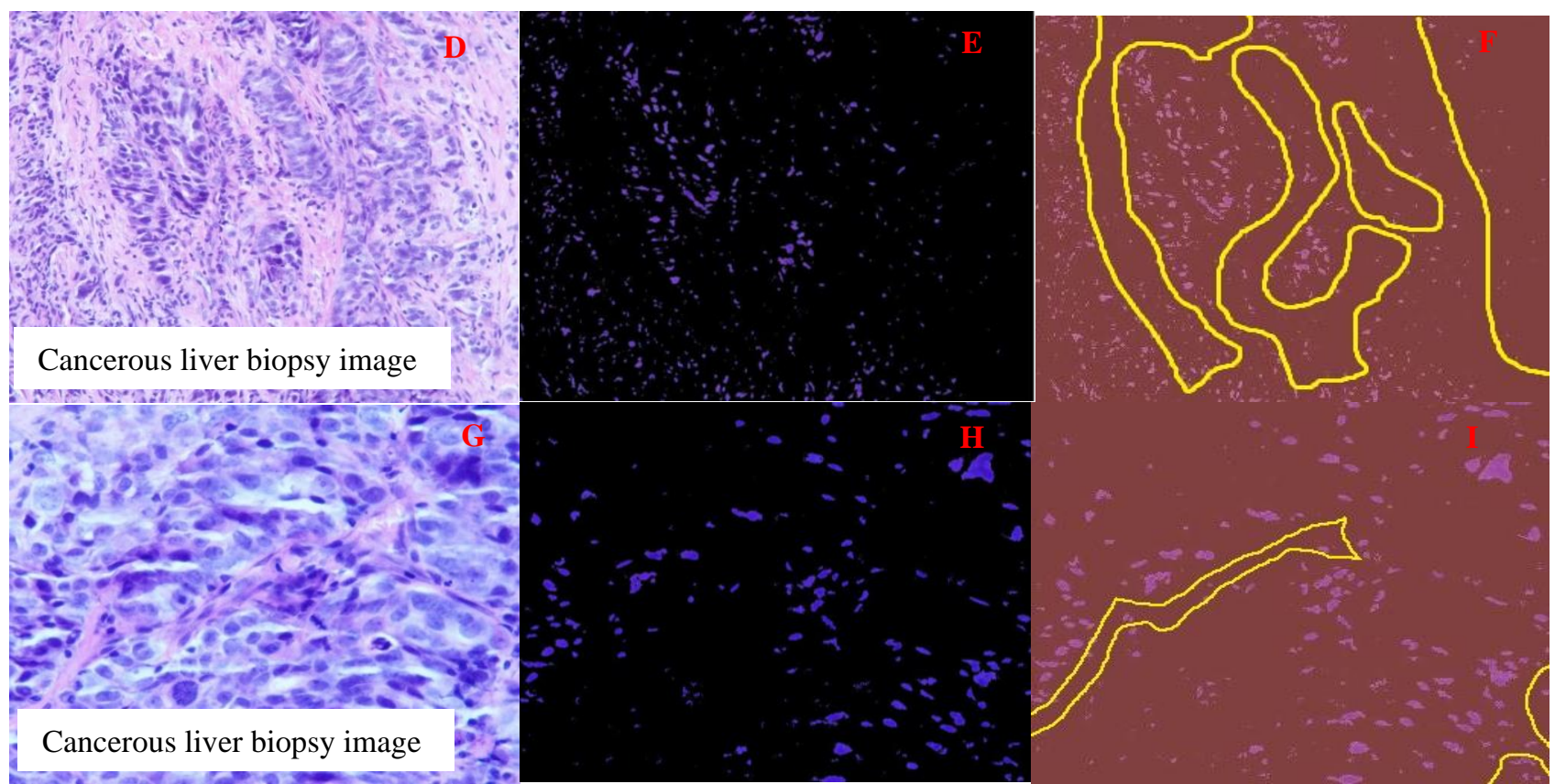

Figure 3. Representative images of liver biopsy samples: A, D, G - original images of Hematoxylin and Eosin stained biopsies; B, E, H- images obtained by K-means clustering approach; C, F, I - images with the highlighted cancerous/noncancerous areas (yellow lines depict cancerous regions detected by MMF).

As it can be observed in Figs. 3F, I, the applied metamaterial approach allows to perform a precise segmentation and classification of the cells composing the biopsy. Doing so, the errors occurred at the image digitization stage fulfilled by applying K-means clustering approach can be easily detected by the MMF. The healthy areas in Fig. 3F, I are marked by the yellow areas.

\section{CONCLUSIONS}

Here we accustomed MMF approach for automated and comprehensive cancer cell detection in biopsy images with high proximity to be used further for clinical diagnostics. This advanced approach allows to simplify the image digitization procedures since there is no need for the precise recognition of the cancerous cells at early stages of the data analysis. Then cancer cell recognition itself is performed by estimation of the cells permittivity with consequently applied effective medium approximation which considers properties of the whole image as a disordered metamaterial model. The main advantage of the proposed technique is its capability to detect the scale of the tumour development giving a powerful diagnostic tool for fighting cancer clinicians. The described formalism can serve as identification of tumour locus, helping in decision making on cancer propagation level in the sample.

\section{ACKNOWLEDGEMENT}

This project has received funding from the European Union's Horizon 2020 research and innovation programme under the Marie Sklodowska Curie grant agreement No 713694 and from Engineering and Physical Sciences Research Council (EPSRC) (Grant No. EP/R024898/1). E.U.R. also acknowledges partial support from the Academic Excellence Project 5-100 proposed by Peter the Great St. Petersburg Polytechnic University.
Authors also acknowledge support from the Russian Science Foundation (Grants \# 18-15-00172).

\section{REFERENCES}

1. R. L. Siegel, K. D. Miller, and A. Jemal, "Cancer Statistics, 2015," CA Cancer J. Clin., vol. 65(1), pp. 5-29, 2015.

2. A. Kaczmarek, P. Vandenabeele, and D. V. Krysko, "Necroptosis: The Release of Damage-Associated Molecular Patterns and Its Physiological Relevance," Immunity, vol. 38(2), pp. 209-223, 2013.

3. A. Kamal, S. Faazil, and M. S. Malik, "Apoptosis-inducing agents: A patent review (2010 - 2013)," Expert Opin. Ther. at., vol. 24(3), pp. 339354, 2014.

4. B. Levine and J. Yuan, "Autophagy in cell death: An innocent convict?" J. Clin. Invest., vol, 115(10), pp. 2679-2688, 2005.

5. S. Bhakdi, P. Thaicharoen, "Easy employment and crosstalk-free detection of seven fluorophores in a widefield fluorescence microscope," Methods Protoc., vol. 1, pp. 20, 2018.

6. E. Meijering, A. E. Carpenter, H. Peng, F. A. Hamprecht, J.-C. OlivoMarin, "Imagining the future of bioimage analysis," Nat. Biotechnol., vol. 34, pp. 1250-1255, 2016.

7. T. Gric, M. Cada, "Analytic solution to field distribution in onedimensional inhomogeneous media," Optics Communications, vol. 322, pp. 183-187, 2014.

8. T. Gric, O. Hess, "Controlling hybrid-polarization surface plasmon polaritons in dielectric-transparent conducting oxides metamaterials via their effective properties," Journal of Applied Physics, vol. 122, pp. 193105, 2017.

9. T. Gric, O. Hess, "Tunable surface waves at the interface separating different graphene-dielectric composite hyperbolic metamaterials," Opt. Express, vol. 25, pp. 11466-11476, 2017.

10. Z. Zhang, H. Ding, X. Yan, L. Liang, D. Wei, M. Wang, Q. Yang, and J. Yao, "Sensitive detection of cancer cell apoptosis based on the nonbianisotropic metamaterials biosensors in terahertz frequency," Opt. Mater. Express, vol. 8, pp. 659-667, 2018.

11. M. Zhu, L. Zhang, S. Ma, J. Wang, J. Su and A. Liu, "Terahertz metamaterial designs for capturing and detecting circulating tumor cells," Mater. Res. Express, vol. 6, pp. 045805, 2019. 
12. A. Khokhlova, I. Zolotovskii, S. Sokolovski, Y. Saenko, E. Rafailov, D. Stoliarov, E. Pogodina, V. Svetukhin, V. Sibirny, A. Fotiadi, “The lightoxygen effect in biological cells enhanced by highly localized surface plasmon-polaritons," Scientific Reports, vol. 9, pp. 18435, 2019.

13. J. Gollub, "Characterizing the effects of disorder in metamaterial structures," Appl. Phys. Lett., vol. 91, pp. 162907, 2007.

14. M. V. Gorkunov, S. A. Grdeskul, I. V. Shadrivov, Y. S. Kivshar, "Effect of microscopic disorder on magnetic properties of metamaterials," Phys. Rev. E Stat. Nonlin. Soft. Matter. Phys., vol. 73(5 Pt 2), pp. 056605, 2006.

15. C. Helgert, C. Rockstuhl, C. Etrich, E.-B. Kley, A. Tünnermann, F. Lederer, T. Pertsch, "Effects of anisotropic disorder in an optical metamaterial," Appl. Phys. A, vol. 103, pp. 591-595, 2011.

16. J. Leroy, C. Dalmay, A. Landoulsi, F. Hjeij, C. Mélin, B. Bessette, C. Bounaix Morand du Puch, S. Giraud, C. Lautrette, S. Battu, F. Lalloué, M. O. Jauberteau, A. Bessaudou, P. Blondy, A. Pothier, "Microfluidic biosensors for microwave dielectric spectroscopy," Sensors and Actuators, A: Physical, vol. 229, pp. 172-181, 2015.

17. G. Li and X.-F. Pang, "Effects of electromagnetic field exposure on electromagnetic properties of biological tissues," Progress in Biochemistry and Biophysics, vol. 38(7), pp. 604-610, 2011.

18. L. Shen, L. R. Margolies, J. H. Rothstein, E. Fluder, R. McBride, W. Sieh, "Deep Learning to Improve Breast Cancer Detection on Screening Mammography," Sci Rep., vol. 9, pp. 12495, 2019.

19. E. Callaway, D. Castelvecchi, D. Cyranoski, E. Gibney, H. Ledford, J. J. Lee, L. Morello, N. Phillips, Q. Schiermeier, J. Tollefson, A. Witze, "2017 in news: the science events that shaped the year," Nature, vol. 552, pp. 304-307, 2017.

20. A. E. Fetit, J. Novak, D. Rodriguez, D. P. Auer, C. A. Clark, R. G. Grundy, A. C. Peet, T. N. Arvanitis, "Radiomics in paediatric neurooncology: a multicentre study on MRI texture analysis," NMR Biomed, vol. 31, pp. 1-13, 2018.

21. S. Goldenberg, G. Nir, S. E. Salcudean, "A new era: artificial intelligence and machine learning in prostate cancer," Nat. Rev. Urol., vol. 16, pp. 391-403, 2019

22. I. Banerjee, A. Crawley, M. Bhethanabotla, H. E. Daldrup-Link, D. L. Rubin, "Transfer learning on fused multiparametric MR images for classifying histopathological subtypes of rhabdomyosarcoma," Comput Med Imaging Graph, vol. 65, pp. 167-175, 2018.

23. O. Kidwai, S. V. Zhukovsky, and J. E. Sipe, "Effective-medium approach to planar multilayer hyperbolic metamaterials: Strengths and limitations," Phys. Rev. A, vol. 85, pp. 053842, 2012.

24. P. Zakharov, F. Dewarrat, A. Caduff, and M. S. Talary, "The effect of blood content on the optical and dielectric skin properties," Physiological Measurement, vol. 32(1), pp. 131-149, 2011.

25. M.-N. Wu, C.-C. Lin, C.-C. Chang, "Brain Tumor Detection Using ColorBased K-Means Clustering Segmentation," IIH-MSP '07: Proceedings of the Third International Conference on International Information Hiding and Multimedia Signal Processing (IIH-MSP 2007), vol. 2, pp. 245-250, 2007.

26. M. Rakesh, T. Ravi, "Image Segmentation and Detection of Tumor Objects in MR Brain Images Using FUZZY C-MEANS (FCM) Algorithm," IJERA, vol. 2(3), pp. 2088-2094, 2012.

27. C. Christine, F. Thomas, "A Study of Efficiency and Accuracy in the Transformation from RGB to CIELAB Color Space," IEEE Transactions on Image Processing, vol. 6(7), pp. 1046-1048, 1997.

28. P. Salembier, J. Serra, "Flat zones filtering, connected operators, and filters by reconstruction," IEEE Trans. Image Process., vol. 4(8), pp. 1153-1160, 1995.

29. H. Sun, J. Yang, M. Ren, "A fast watershed algorithm based on chain code and its application in image segmentation," Pattern Recogn. Lett., vol. 26(9), pp. 1266-1274, 2005.

30. G. Bradski, The OpenCV Library. Dr. Dobb's J. Softw. Tools, 2000.

31. N. Otsu, "A threshold selection method from gray-level histograms," IEEE transactions on systems, man, cybernetics, vol. 9, pp. 62-66, 1979.
32. S. Bhakdi, P. Thaicharoen, "Easy employment and crosstalk-free detection of seven fluorophores in a widefield fluorescence microscope," Methods Protoc., vol. 1, pp. 20, 2018.

33. E. Meijering, A. E. Carpenter, H. Peng, F. A. Hamprecht, J.-C. OlivoMarin, "Imagining the future of bioimage analysis," Nat. Biotechnol., vol. 34, pp. 1250-1255, 2016.

34. O. Kidwai, S. V. Zhukovsky, and J. E. Sipe, "Effective-medium approach to planar multilayer hyperbolic metamaterials: Strengths and limitations," Phys. Rev. A, vol. 85, pp. 053842, 2012.

35. Tatjana Gric, Sergei G. Sokolovski, Nikita Navolokin, Oksana Glushkobskaya, and Edik U. Rafailov, "Metamaterial formalism approach for advancing the recognition of glioma areas in brain tissue biopsies," Opt. Mater. Express, vol. 10, pp. 1607-1615, 2020.

36. P. Zakharov, F. Dewarrat, A. Caduff, and M. S. Talary, "The effect of blood content on the optical and dielectric skin properties," Physiological Measurement, vol. 32(1), pp. 131-149, 2011.

37. T. H. Tjia, P. Bordewijk, and C. J. F. B"ottcher, "On the notion of dielectric friction in the theory of dielectric relaxation," Advances in Molecular Relaxation Processes, vol. 6(1), pp. 19-28, 1974.

38. J. C. Maxwell-Garnett, "Colours in metal glasses and films," Philosophical Transactions of the Royal Society A Mathematical Physical \& Engineering Sciences, vol. 203, pp. 385-420 (1904).

39. M. Bruggerman, W. Kalkner, A. Campus, and A. Smedberg, "Electrochemical effects at the conductor/dielectric interface-a description of the mechanism," IEEE International Conference on Solid Dielectrics, vol. 1, pp. 383-386, 2004.

40. T. Hanai, "A remark on theory of dielectric dispersion due to interfacial polarization," Colloid Polymer Science, vol. 175(1), pp. 61-62, 1961.

41. T. H. Tjia, P. Bordewijk, and C. J. F. B"ottcher, "On the notion of dielectric friction in the theory of dielectric relaxation," Advances in Molecular Relaxation Processes, vol. 6(1), pp. 19-28, 1974.

42. S. E. Skipetrov, "Effective dielectric function of a random medium," Physical Review B-Condensed Matter and Materials Physics, vol. 60(18), pp. 12705-12709, 1999.

43. C. J. F. Bo"ttcher, P. Bordeweijk, Theory of Electric Polarisation, Elsevier, Amsterdam, 1978

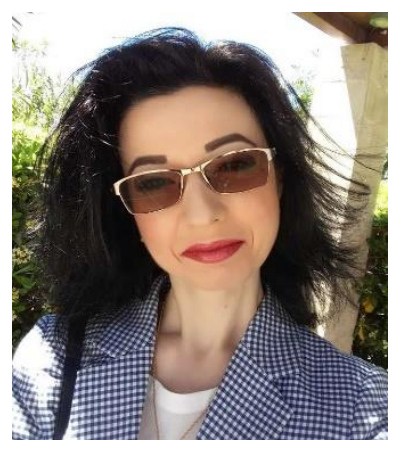

Dr. Gric's research career has been focused on the investigation of waveguide devices (waveguide modulators, filters etc.), namely on proposing their electrodynamical analysis. Applied research includes the design of microwave frequency selective structures, waveguide modulators, filters. Fundamental research is primarily concerned with developing rigorous computational methods for the electrodynamical analysis of the waveguide structures. Another major goal of her studies is plasmonics as the examination of the interaction between electromagnetic field and free electrons in a metal. The optically-active nanostructures have been simulated and their fundamental photonic properties have been explored. Moreover, the broad scope of research carried out by Dr. Gric has included investigations into the new fascinating properties of novel materials. Dr. Gric is involved in development of unusual materials and structures that can manipulate the flow of light in ways that are useful in optical sensing, photovoltaics, 
solid state lighting, fiber optics and other applications. Dr. Gric has published extensively in her field of investigation with more than 50 peer-reviewed papers in top journals in physics, electrodynamics, and optics and has written one book and two book chapters. It is worth noting that her recent publication rate is getting even higher with her being the first author.

Sergei G. Sokolovski received the M.Sc. (Hons.) degree in biochemistry from Byelorussian State University, Belarus, in 1989, and the Ph.D. degree in biophysics from the National Academy of Sciences of Belarus, Belarus, in 1993. In 1995, he was working for leading Europe and U.K. Universities (Jena, Karlsruhe, York, Glasgow, Dundee) researching fundamental processes of the cell photonics, cell stress and hormone signalling, ion channel regulation, membrane proteins trafficking, and genes regulation. In 2008, he was working in developing laser systems bringing his 25 years' experience in studying the fundamental principles of the light perception and stress responses of living organisms to the development of laser based devices for noninvasive diagnostics and disease phototreatments.

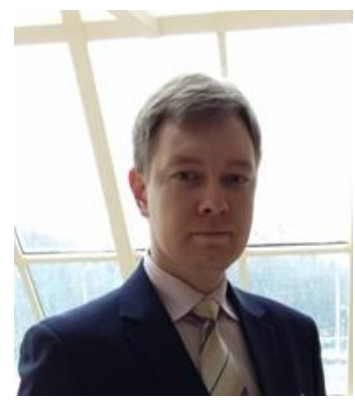

Alekseev Alexander Gennadievich was born on October 21, 1985. In 2009, he graduated with honors from the Medical Institute of Orel State University with a degree in General Medicine. In 2009-2011 he studied in full-time postgraduate studies in the specialty 03.03.04 "Cell Biology, Cytology, Histology". November 24, 2011 by the decision of the Dissertation Council at the Research Institute of Human Morphology RAMS awarded the degree of candidate of medical sciences. October 31, 2017 by order of the Ministry of Education and Science of the Russian Federation awarded the academic title of assistant professor in the specialty "Human Anatomy". In 2012-2018, he worked as an assistant professor at the Department of Anatomy, Surgery and Disaster Medicine, Federal State Budgetary Educational Establishment of Higher Education "Orel State University named after I.S. Turgenev". In 2018, he was elected head of the department. Since February 2020, Dean of the Faculty of Medicine, Medical Institute of OSU named after I.S. Turgenev. Pathologist. Clinical laboratory diagnostics doctor. Candidate of Medical Sciences, Associate Professor. The author of more than 80 published scientific papers; co-authored four patents and a monograph. Research interests: general laws of morphogenesis, embryogenesis and ontogenesis of humans and animals, clinical morphology, fluorescence diagnostics of biological tissues in vivo and in vitro.

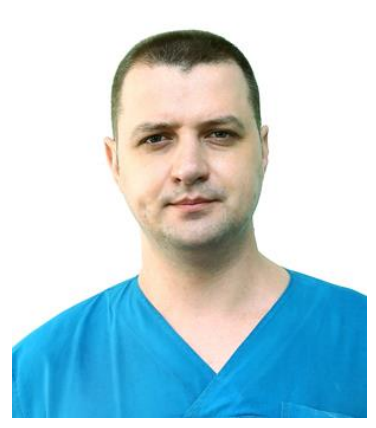

Andrian Mamoshin was born in Orel, USSR, in 1978. He received M.Sc. and M.D degrees from the Smolensk State Medical University, Smolensk, Russia, in 2001 and 2005, respectively. Since 2003, he is a surgeon of the Orel Regional Clinical Hospital, Orel, Russia. In 2018, he became the main medical collaborator of the Russian Science Foundation project 18-15-00201 "Clinical and experimental substantiation of multiparametric optical biopsy of hepatopancreatoduodenal zone organs for minimally invasive surgical operations". Since 2018, he is a senior researcher at Research and Development Center of Biomedical Photonics, Orel State University, Orel, Russia. His research interests include development and implementation of optical biopsy techniques in minimally invasive surgery of abdominal organs; controlled minimally invasive interventions in abdominal surgery; implementation of modern ultrasound examination methods in abdominal surgery. He is the author of 7 papers in peer-reviewed journals, 13 study guides and monographs, and 5 patents.

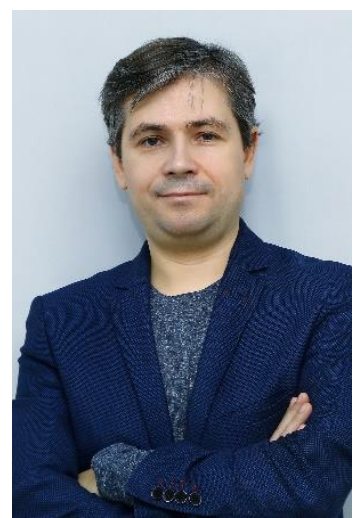

Andrey Dunaev was born in Kishinev, USSR, in 1976. He received his $\mathrm{MSc}$ and $\mathrm{PhD}$ degrees in Instrument Engineering from the Orel State University, Orel, Russia, in 1999 and 2002, respectively. From 2005 to 2011 he was an Associate Professor in the Department of "Instrument Engineering, metrology and certification", Orel State University, Orel, Russia. From 2011 to 2013 he was a Postdoctoral Researcher (Marie Currie Research Fellow) in the Photonics and Nanoscience Group, University of Dundee, Dundee, UK. Since 2014 he is the head at the Research and Development Center of Biomedical Photonics, Orel State University, Orel, Russia. His research interests are devoted to metrological, methodological and instrumentation support of optical noninvasive diagnostics (Laser Doppler Flowmetry, Fluorescence Spectroscopy, Diffuse Reflectance Spectroscopy, etc.) and include development multiparameter optical diagnostics of microcirculatory-tissue systems. He is the author of 3 books, more than 50 papers in peer-reviewed journals and more than 10 patents. 


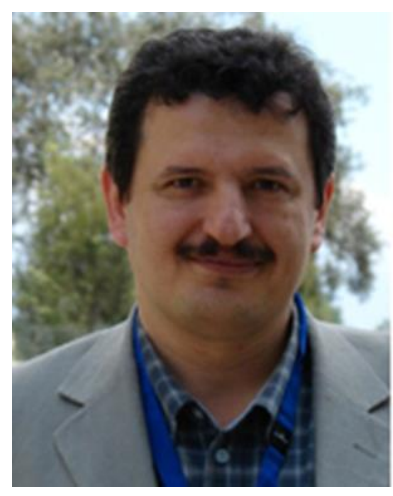

Edik Rafailov (SM'05) received the Ph.D. degree from the Ioffe Institute in 1992. In 1997 he moved to St Andrews University (UK) and 2005, he established a new group in the Dundee University. In 2014 he and his Optoelectronics and Biomedical Photonics Group moved to the Aston University (UK). He has authored and co-authored over 450 articles in refereed journals and conference proceedings, including two books (WILEY), ten invited chapters and numerous invited talks. Prof. Rafailov coordinated the $€ 14.7 \mathrm{M}$ FP7 FAST-DOT project development of new ultrafast lasers for Biophotonics applications and the $€ 12.5 \mathrm{M}$ NEWLED project which aims to develop a new generation of white LEDs. Currently he coordinated the H2020 FET Mesa-Brain (€3.3M, aims to develop 3D nano-printing technology for functional three-dimensional human stem cell derived neural networks), NEUROPA ( $€ 3.6 \mathrm{M}$, aims to develop novel non-invasive theranostic approachs), and EPSRC proposal (£1M, compact $\mathrm{THz}$ based systems for neuroscience applications). He also leads a few others projects funded by EU FP7, H2020 and EPSRC (UK). His current research interests include high-power $\mathrm{CW}$ and ultrashort-pulse lasers; generation of UV/visible/IR/MIR and THz radiation, nanostructures; nonlinear and integrated optics; biomedical photonics. 